\title{
Non-coding recurrent mutations in chronic lymphocytic leukaemia
}

Xose S. Puente, Silvia Beà, Rafael Valdés-Mas, Neus Villamor, Jesús Gutiérrez-Abril, José I. Martín-Subero, Marta Munar, Carlota Rubio-Pérez, Pedro Jares, Marta Aymerich, Tycho Baumann, Renée Beekman, Elías Campo* \& Carlos López-Otín† et al.

\section{PAPER ABSTRACT}

Chronic lymphocytic leukaemia (CLL) is a frequent disease in which the genetic alterations determining the clinicobiological behaviour are not fully understood. Here we describe a comprehensive evaluation of the genomic landscape of 452 CLL cases and 54 patients with monoclonal B-lymphocytosis, a precursor disorder. We extend the number of CLL driver alterations, including changes in ZNF292, ZMYM3, ARID1A and PTPN11. We also identify novel recurrent mutations in non-coding regions, including the 3 region of $\mathrm{NOTCH1}$, which cause aberrant splicing events, increase NOTCH1 activity and result in a more aggressive disease. In addition, mutations in an enhancer located on chromosome 9p13 result in reduced expression of the B-cell-specific transcription factor PAX5. The accumulative number of driver alterations $(0$ to $\geq 4)$ discriminated between patients with differences in clinical behaviour. This study provides an integrated portrait of the CLL genomic landscape, identifies new recurrent driver mutations of the disease, and suggests clinical interventions that may improve the management of this neoplasia.

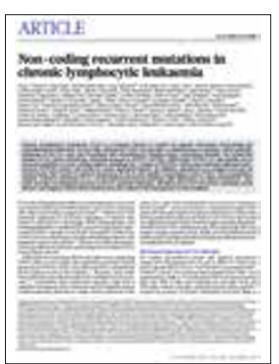

Citation: Nature 526, 519-524 (2015)

doi: $10.1038 /$ nature 14666

- RECEIVED 23 February 2015

- ACCEPTED 15 June 2015

- PUBLISHED ONLINE 22 July 2015

\section{SUMMARY}

Insights from the chronic lymphocytic leukaemia (CLL) genome landscape. Sequencing more than 500 cases of CLL has uncovered most of the genes that are frequently mutated in this pathology and involved in tumour initiation and progression. In addition, whole-genome sequencing has allowed the identification of functional mutations in non-coding regions with a clear effect on disease prognosis.

\section{The question}

Tumour transformation occurs as a result of the accumulation of mutations in the genome of healthy cells. Mutations that lead to the dysregulation of important cellular processes and contribute to tumour transformation are known as driver mutations ${ }^{1,2}$. The identification of driver mutations is an important step towards understanding the molecular mechanisms of cancer. It also represents an opportunity to identify novel therapeutic targets, and provides key information for prognosis and patient stratification. However, for most tumour types, the genomic alterations involved in development and progression are poorly known. The unbiased identification of driver mutations has been limited by the high costs of genome sequencing. During the past decade, however, unprecedented advances in DNA-sequencing technologies have overcome this limitation, allowing the comprehensive study of tumour genomes ${ }^{3}$. The clinical heterogeneity and limited knowledge of the genomic alterations that cause CLL prompted the need for an integrated analysis of these parameters and their clinical significance.

\section{The solution}

We have performed the largest sequencing study in a tumour type by analysing 506 CLL cases, including 150 that were investigated by wholegenome sequencing (see Graphical abstract). The tumour genome was sequenced from DNA obtained from tumour cells, and the normal genome was sequenced from non-tumour cells from the same patient. The genomes were compared to identify somatic mutations - those present in the tumour genome but not in normal DNA. We found an average of 2,400 mutations per tumour, of which 13 affected proteincoding genes. By comparing the frequency at which each gene was mutated in the 506 patients, we identified 60 genes that were mutated at a higher frequency than expected by chance, representing the most common driver genes in CLL. In addition, we found recurrent mutations in non-coding regions of the genome that result in a functional effect that is important for tumour growth. More than $2 \%$ of the tumours harboured the same mutation in the $3^{\prime}$-untranslated region of the gene NOTCH1. This mutation creates an acceptor site leading to aberrant splicing in the last exon. This results in a protein that lacks the C-terminal PEST 
domain, leading to a more stable protein and accumulation of mutated NOTCH1 in the nucleus of tumour cells. We also found mutations in an enhancer, leading to reduced expression of the B-cell transcription factor PAX5. These non-coding mutations alter the activity of transcription factors that are of known importance in B-cell development.

\section{The implications}

This research provides an overall picture of the mutations that drive oncogenic transformation in CLL. The study shows that CLL constitutes a highly heterogeneous disease from a genomic point of view, which might explain the different courses observed in the clinic $^{4-6}$. More than $90 \%$ of tumours contain at least one of the driver mutations uncovered in this study, and there is a clear relationship between the total number of driver mutations present per tumour and prognosis. The low frequency at which most of these mutated drivers appear makes evaluating putative associations with clinicopathological features difficult ${ }^{7}$. However, this work provides the basis for exploring the clinical impact of the molecular heterogeneity of the disease in independent cohorts. By reducing the number of genomic alterations thought to be involved in CLL to less than 60, this information will facilitate further studies investigating the relationship between particular mutations and response to treatment. Together, these studies will contribute to the generation of a body of data that will sustain ongoing precision-medicine initiatives for cancer treatment.

\section{, \\ The full version of this article is at Nature's website at go.nature.com/28p2tuc}

\section{GRAPHICAL ABSTRACT}
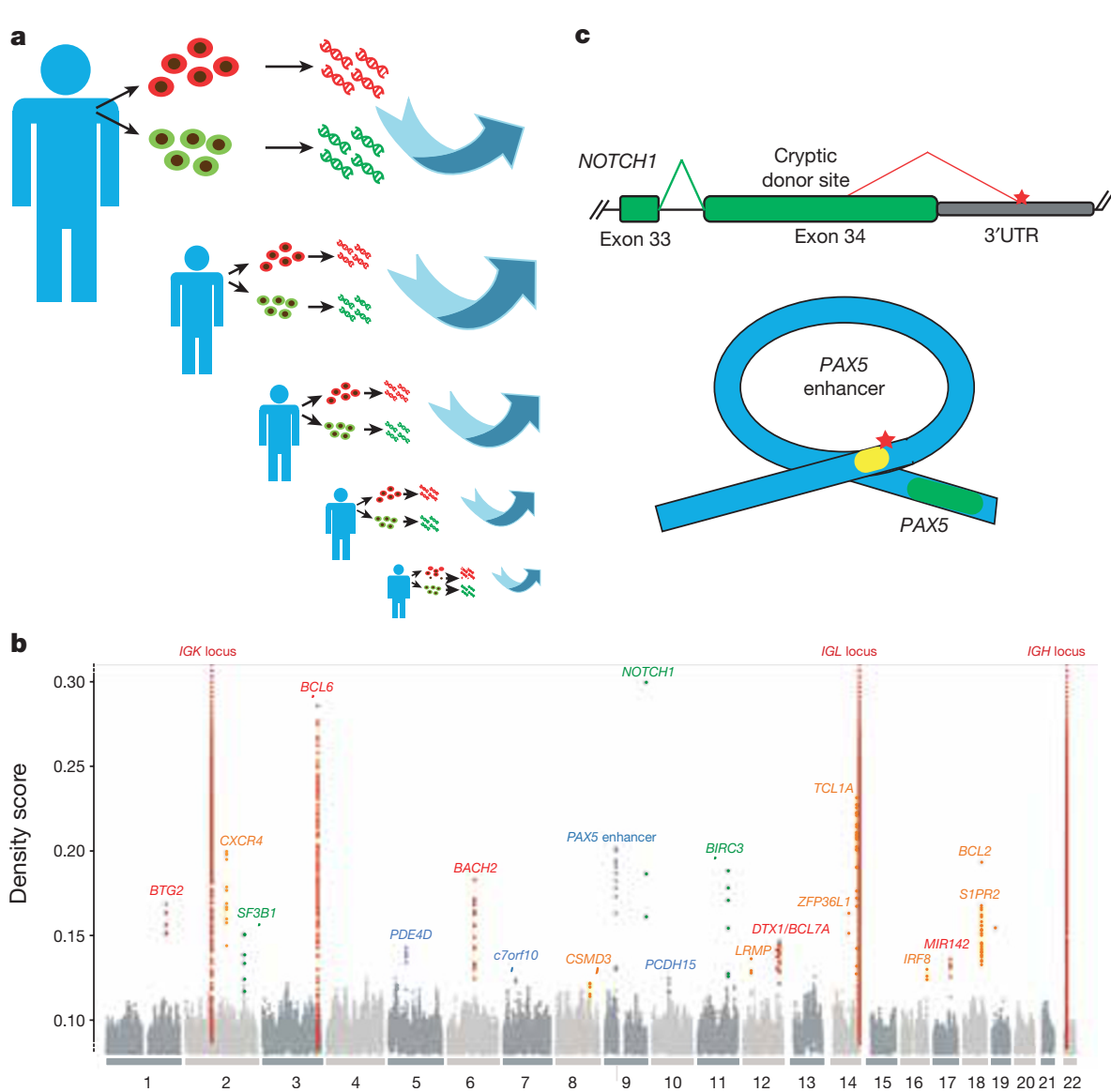

Integrated genomic analysis of CLL. a, Genomes from tumour and non-tumour cells from the same patient were sequenced for more than 500 CLL cases to identify somatic mutations. $\mathbf{b}$, Combined analysis of the somatic mutations allowed the identification of genomic regions mutated at a higher density in CLL than expected by chance. c, Non-coding mutations in the $3^{\prime}$-untranslated region (UTR) of NOTCH1 and in an enhancer of $P A X 5$ result in aberrant splicing and altered expression of these genes, respectively.

\section{FURTHER READING}

1. Stratton, M. R., Campbell, P. J. \& Futreal, P. A. The cancer genome. Nature 458, 719-724 (2009).

The concept of the cancer genome and driver genes is defined in this study.

2. The International Cancer Genome Consortium International network of cancer genome projects. Nature 464, 993-998 (2010). This paper describes the scope and structure of the International Cancer Genome Consortium.

3. Puente, X. S. et al. Whole-genome sequencing identifies recurrent mutations in chronic lymphocytic leukaemia. Nature 475, 101-105 (2011).

In this study the first four CLL genomes are sequenced, with the identification of four novel oncogenes mutated in this cancer type, including NOTCH1.

4. Rossi, D. et al. Mutations of NOTCH1 are an independent predictor of survival in chronic lymphocytic leukemia. Blood 119, 521-529 (2012).

This study confirmed that the mutations in NOTCH1 identified by whole-genome sequencing constitute an independent predictor of overall survival in CLL, identifying cases with a poor prognosis.

5. Quesada, V. et al. Exome sequencing identifies recurrent mutations of the splicing factor SF3B1 gene in chronic lymphocytic leukemia. Nature Genet. 44, 47-52 (2012).

This study and ref. 6 provide the first comprehensive analysis of genes mutated in CLL by exome sequencing, with the identification of genes recurrently mutated, including the splicing factor gene SF3B1.

6. Wang, L. et al. SF3B1 and other novel cancer genes in chronic lymphocytic leukemia. N. Engl. J. Med. 365, 2497-2506 (2011). See ref. 5 .

7. Landau, D. A. et al. Evolution and impact of subclonal mutations in chronic lymphocytic leukemia. Cell 152, 714-726 (2013).

This study showed that certain genes, such as TP53, SF3B1 and NRAS, are mutated in minor clones of CLL tumours, but are mainly responsible for the major clone that arises at relapse.

\section{RELATED CONTENT}

\author{
Cancer Genomes: Chronic Lymphocytic \\ Leukemia \\ Universidad de Oviedo \\ go.nature.com/28sryxp
}

Role of non-coding sequence variants in cancer E. Khurana et al.

go.nature.com/28npzkw 